\title{
Anomalous Hall effect in a two dimensional electron gas with magnetic impurities
}

\author{
Tamara S. Nunner, ${ }^{1}$ Gergely Zaránd, ${ }^{2}$ and Felix von Oppen ${ }^{1}$ \\ ${ }^{1}$ Institut für Theoretische Physik, Freie Universität Berlin, Arnimallee 14, 14195 Berlin, Germany \\ ${ }^{2}$ Institute of Physics, Technical University Budapest, Budapest, H-1521, Hungary
}

(Dated: November 10, 2018)

\begin{abstract}
Magnetic impurities play an important role in many spintronics-related materials. Motivated by this fact, we study the anomalous Hall effect in the presence of magnetic impurities, focusing on two-dimensional electron systems with Rashba spin-orbit coupling. We find a highly nonlinear dependence on the impurity polarization, including possible sign changes. At small impurity magnetizations, this is a consequence of the remarkable result that the linear term is independent of the spin-orbit coupling strength. Near saturation of the impurity spins, the anomalous Hall conductivity can be resonantly enhanced, due to interference between potential and magnetic scattering.
\end{abstract}

PACS numbers: 72.15.Gd,72.20.Dp,72.25.-b

Introduction.-In ferromagnetic materials, the Hall resistance acquires an anomalous contribution which is proportional to the magnetization of the sample [1, 2, 3]. Although this anomalous Hall effect (AHE) has become a standard tool to determine the magnetization of ferromagnets and has been known for more than a century, its mechanism is still under debate. The interest in the origin of the AHE has recently been renewed due to its close relation to the spin Hall effect. Particular attention has been paid to intrinsic mechanisms [1], where the spin-orbit interaction modifies the band-structure. This is in contrast to extrinsic mechanisms [2, 3, 4], where the spin-orbit interaction appears only in the impurity potential. Surprising results have been found even for simple systems such as a two-dimensional electron gas (2DEG) with Rashba spin-orbit interaction where it turns out that in the presence of pointlike potential disorder the AHE vanishes when both of the spin-split bands are occupied [6, 7, 8, 9, 10, 11, 12].

So far, most treatments of the intrinsic AHE have only considered the presence of potential impurities. However, in many materials, which are of interest for spintronics applications such as diluted magnetic semiconductors, magnetic impurities play a fundamental role. Although extrinsic mechanisms for the AHE based on scattering by magnetic impurities have been suggested [5], little is known about the influence of magnetic impurities in materials with strong spin-orbit interaction, where the AHE is induced by intrinsic mechanisms. To learn about the role of magnetic impurities, we avoid the complexities of realistic band structures and instead provide a thorough analysis of a simple model: a 2DEG with Rashba spin-orbit interaction where the presence of magnetic impurities is known to induce a finite AHE even when both spin-split bands are occupied [10]. We find that surprisingly rich physics emerges even in this model system.

We expect that our model can be tested experimentally in magnetically doped 2DEGs [13], once they are produced with asymmetric confinement potentials. This may be realized in heterojunctions, made of a material with large spin-orbit coupling. At present, a robust AHE has been observed in a magnetically doped 2DEG with weak spin-orbit coupling, based on a modulation-doped quantum well of $\mathrm{Zn}_{1-x-y} \mathrm{Cd}_{y} \mathrm{Mn}_{x}$ Se $(x \sim 0.02, y \sim 0.12)$ sandwiched between ZnSe barriers [14].

To construct a theory for the AHE in magnetically doped 2DEGs with Rashba spin-orbit coupling, we use the Hamiltonian

$$
H=\frac{\mathbf{p}^{2}}{2 m}+\alpha\left(p_{y} \sigma_{x}-p_{x} \sigma_{y}\right)+\sum_{i}\left[V+J \mathbf{S}_{i} \cdot \boldsymbol{\sigma}\right] \delta\left(\mathbf{r}-\mathbf{R}_{i}\right) .
$$

Here, $\alpha$ denotes the strength of the spin-orbit interaction. The impurities at positions $\mathbf{R}_{i}$ affect the conduction electrons through a potential $V \delta\left(\mathbf{r}-\mathbf{R}_{i}\right)$ and an exchange coupling $J \mathbf{S}_{i} \cdot \boldsymbol{\sigma}$. (Here, $\mathbf{S}_{i}$ denotes the impurity and $\boldsymbol{\sigma}$ the electron spin.) Our analysis below includes interference of amplitudes from potential and magnetic scattering, and accounts for all significant contributions to the AHE, including skew scattering. Remarkably, we find that the AHE deviates significantly from the conventional linear magnetization dependence except for the region of very small polarization of the impurity spins. For strong impurity polarization, we obtain a resonant behavior of the anomalous Hall conductivity. This enhancement arises from an interference-induced suppression of the scattering rate for the minority carriers.

Anomalous Hall conductivity.-Our theory is based on the Streda-Kubo approach which decomposes the Hall conductivity $\sigma_{y x}=\sigma_{y x}^{I}+\sigma_{y x}^{I I}$ into a Fermi-surface contribution $\sigma_{y x}^{I}$ and a contribution $\sigma_{y x}^{I I}$ from the entire Fermi sea [16]. The AHE is dominated by the Fermi surface contribution

$\sigma_{y x}^{I}=\frac{e^{2}}{4 \pi V} \operatorname{Tr}\left[v_{y} G_{\epsilon_{F}}^{R} v_{x}\left(G_{\epsilon_{F}}^{R}-G_{\epsilon_{F}}^{A}\right)-v_{y}\left(G_{\epsilon_{F}}^{R}-G_{\epsilon_{F}}^{A}\right) v_{x} G_{\epsilon_{F}}^{A}\right]$,

where $G^{R, A}$ denotes the advanced and retarded Green functions and the velocity operators are given by $v_{x, y}=$ $p_{x, y} / m \mp \alpha \sigma_{y, x}$. It can be shown that the contributions of $G^{R} G^{R}$ and $G^{A} G^{A}$ are of higher order in the disorder scattering rate $\Gamma$ [15], leaving only the mixed $G^{R} G^{A}$ 
terms to be considered. Regarding $\sigma_{y x}^{I I}$ it is known that it vanishes for $\epsilon_{F}>h$ in clean systems [12], where $h$ is the effective magnetic field. This remains true also in the presence of magnetic impurities as long as vertex corrections are neglected, which contribute only to higher order in $\Gamma$ 15].

To compute the anomalous Hall conductivity, we treat the exchange interaction at a mean-field level, average over the impurity potential, and incorporate disordereffects perturbatively. Within the mean field approximation, the exchange interaction leads to an additional internal Zeeman field acting on the electrons, which is typically larger than the applied magnetic field $h_{\text {ext }}$. Thus, the electrons are subject to an average effective Zeeman field $h \simeq \epsilon_{J}\left\langle S_{z}\right\rangle / S$, where $\epsilon_{J}=n_{i} J S$ is the maximal exchange field and $n_{i}$ denotes the concentration of impurities. At mean-field level, the nonzero impurity-spin expectation values are given by $\left\langle S_{z}\right\rangle=-\partial \ln Z / \partial(\beta \tilde{h})$, $\left\langle S_{z}^{2}\right\rangle=(1 / Z) \partial^{2} Z / \partial(\beta \tilde{h})^{2}$ in terms of the Brillouin partition function $Z=\sinh (\beta J \tilde{h}(2 S+1) / 2) / \sinh (\beta \tilde{h} / 2)$ and the effective field $\tilde{h}=n J\langle\sigma\rangle$ acting on the impurity spins. Here, $n$ and $\langle\sigma\rangle$ denote the density and polarization of the electrons. We shall not determine $\left\langle S_{z}\right\rangle$ self-consistently. Instead, we treat it as a parameter which also determines the impurity spin fluctuation $\left\langle S_{z}^{2}\right\rangle$ within this mean field approach.

Let us now consider the effects of disorder. In the mean field approximation, the dispersion of the two subbands is given by $E_{k, \pm}=k^{2} / 2 m \pm \lambda_{k}$ with $\lambda_{k}=\sqrt{h^{2}+\alpha^{2} k^{2}}$ and the retarded Green function takes the form $G^{0, R}=$ $G_{0}^{0, R}+\sum_{i=x, y, z} G_{i}^{0, R} \sigma_{i}$ with

$$
\begin{aligned}
G_{0}^{0, R} & =\frac{1}{2}\left(G_{+}^{0, R}+G_{-}^{0, R}\right), G_{z}^{0, R}=-\frac{h}{2 \lambda_{p}}\left(G_{+}^{0, R}-G_{-}^{0, R}\right) \\
G_{x / y}^{0, R} & = \pm \frac{\alpha p_{y / x}}{2 \lambda_{p}}\left(G_{+}^{0, R}-G_{-}^{0, R}\right), G_{ \pm}^{0, R}=\frac{1}{\omega-E_{p \pm}+i 0^{+}} .
\end{aligned}
$$

Deriving the retarded self-energy of the 2DEG within the Born approximation, we obtain $\Sigma^{R}=-i\left(\Gamma+\Gamma_{z} \sigma_{z}\right)$ with

$$
\begin{aligned}
& \Sigma^{R}=-\frac{i n_{i}}{4}\left\{\left[V^{2}+J^{2} S(S+1)+2 V J\left\langle S_{z}\right\rangle \sigma_{z}\right] K_{1}\right. \\
& \left.-\left[2 V J\left\langle S_{z}\right\rangle+\left[V^{2}+J^{2}\left(2\left\langle S_{z}^{2}\right\rangle-S(S+1)\right)\right] \sigma_{z}\right] h K_{2}\right\} .
\end{aligned}
$$

Here, $K_{1}=\left(\nu_{+}+\nu_{-}\right)$and $K_{2}=\nu_{+} / \lambda_{+}-\nu_{-} / \lambda_{-}$where $\nu_{ \pm}=m \lambda_{ \pm} / \sqrt{h^{2}+2 \epsilon_{\mathrm{so}} \epsilon_{F}+\left(\epsilon_{\mathrm{so}}\right)^{2}}$, where $\epsilon_{\mathrm{so}}=\alpha^{2} m$ is related to the density of states of the two bands at the Fermi energy $\epsilon_{F}$ and $\lambda_{ \pm}=\lambda_{k_{ \pm}}$with $k_{ \pm}$being the corresponding Fermi wavevectors. If only pointlike potential scatterers are present and scattering from magnetic impurities is neglected, the anomalous Hall conductivity vanishes except for the extreme limit $\epsilon_{F}<h$. In this paper, we focus on the more realistic regime of $\epsilon_{F}>h$ where both subbands are partially occupied and where it is the magnetic impurities which induce a nonzero anomalous Hall effect.

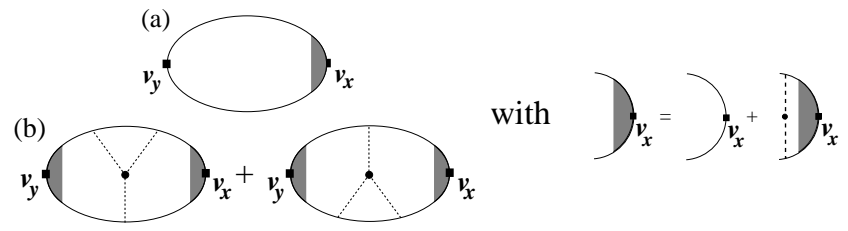

FIG. 1: Anomalous Hall conductivity: (a) Ladder contribution and (b) skew scattering contribution, in analogy to Refs. [11, 12] we only consider diagrams with a single third order impurity vertex with both external current vertices renormalized by ladder type vertex corrections.

When both subbands are occupied, one finds $K_{2}=0$ and hence a significant simplification of the self energy in Eq. (5). The impurity averaged Green function also takes the form of Eq. (3), with the replacements $E \rightarrow E+i \Gamma$ and $h \rightarrow h+i \Gamma_{z}$. In the following, we assume weak disorder scattering in the sense $\Gamma_{z} \ll \lambda_{p}$ such that

$$
G_{ \pm}^{R} \simeq \frac{1}{\omega-E_{p \pm}+i \Gamma_{ \pm}}, \quad \Gamma_{ \pm}=\Gamma \mp \Gamma_{z} \frac{h}{\lambda_{ \pm}} .
$$

The anomalous Hall conductivity $\sigma_{y x}^{I}$ can now be computed as the sum of the ladder diagrams $\sigma_{y x}^{I, l}$ (see Fig. 1(a)) and the skew-scattering contribution $\sigma_{y x}^{I, s}$ (see Fig. 1(b)). Using $n_{i} \tilde{V}^{2}=n_{i}\left(V^{2}-J^{2}\left\langle S_{z}^{2}\right\rangle\right)$, a lengthy but standard evaluation of the diagrams yields

$$
\sigma_{y x}^{I}=\sigma_{y x}^{I, l}+\sigma_{y x}^{I, s}
$$

with

$$
\begin{aligned}
\sigma_{y x}^{I, l} & =\frac{e^{2}}{2 \pi} 2 \alpha\left(\gamma_{1}+i I_{3}\right) \\
& -\frac{e^{2}}{\pi} \frac{n_{i} \tilde{V}^{2}\left(2 \gamma_{1} \gamma_{2}\left(1-n_{i} \tilde{V}^{2} I_{1}\right)+i n_{i} \tilde{V}^{2} I_{2}\left(\gamma_{2}^{2}-\gamma_{1}^{2}\right)\right)}{\left(1-n_{i} \tilde{V}^{2} I_{1}\right)^{2}-\left(n_{i} \tilde{V}^{2} I_{2}\right)^{2}} \\
\sigma_{y x}^{I, s} & =\frac{e^{2}}{2 \pi} \frac{2 n_{i} m J\left\langle S_{z}\right\rangle\left(V^{2}-J^{2} S(S+1)\right) \gamma_{2}^{2}}{\left(1-n_{i} \tilde{V}^{2} I_{1}\right)^{2}}
\end{aligned}
$$

where

$$
\gamma_{1}=i\left(I_{3}-\alpha I_{2}\right) \quad \gamma_{2}=I_{4}-\alpha I_{1}
$$

and

$$
\begin{aligned}
& I_{1} \approx \frac{1}{8}\left(\left(1-\frac{h^{2}}{\lambda_{+}^{2}}\right) \frac{\nu_{+}}{\Gamma_{+}}+\left(1-\frac{h^{2}}{\lambda_{-}^{2}}\right) \frac{\nu_{-}}{\Gamma_{-}}\right) \\
& I_{2} \approx-\frac{i}{4}\left(\frac{\nu_{+} h}{\lambda_{+}^{2}}+\frac{\nu_{-} h}{\lambda_{-}^{2}}-\frac{\Gamma_{z}}{\Gamma_{+}} \frac{\nu_{+} \alpha^{2} k_{+}^{2}}{\lambda_{+}^{3}}+\frac{\Gamma_{z}}{\Gamma_{-}} \frac{\nu_{-} \alpha^{2} k_{-}^{2}}{\lambda_{-}^{3}}\right) \\
& I_{3} \approx-\frac{i}{4} \alpha \Gamma_{z}\left(\frac{\nu_{+}}{\Gamma_{+} \lambda_{+}}\left(\frac{\epsilon_{F}}{\lambda_{+}}-1\right)+\frac{\nu_{-}}{\Gamma_{-} \lambda_{-}}\left(\frac{\epsilon_{F}}{\lambda_{-}}+1\right)\right) \\
& I_{4} \approx-\frac{1}{4} \alpha\left(\epsilon_{F}\left(\frac{\nu_{+}}{\Gamma_{+} \lambda_{+}}-\frac{\nu_{-}}{\Gamma_{-} \lambda_{-}}\right)-\left(\frac{\nu_{+}}{\Gamma_{+}}+\frac{\nu_{-}}{\Gamma_{-}}\right)\right) .
\end{aligned}
$$

Here we have taken the weak scattering limit of $\sigma_{y x}^{I, s}$. As a result of several cancellations these expressions turn out 
to be formally similar to the ones of Ref. [12], derived for purely potential scatterers. The main differences are that additional terms proportional to $\left\langle S_{z}\right\rangle$ appear in the selfenergy and that $n_{i} V^{2}$ is replaced by $n_{i} \tilde{V}^{2}$ in the vertex corrections. Furthermore, $V^{2}$ is replaced by $V^{2}-J^{2} S(S+$ 1 ) in the numerator of the skew scattering contribution.

We remark that the expression for the ladder contribution reduces to the results of Inoue et al. 10], i.e., $\sigma_{y x}^{I, l} \approx e^{2} \epsilon_{\mathrm{so}} \epsilon_{F}^{2} \delta^{2} /\left(2 \pi h^{3}\right)$ with $\delta=\Gamma_{z} / \Gamma \approx 2 J\left\langle S_{z}\right\rangle / V$, when taking the special limit of small spin-orbit interaction, large Fermi energy $\Delta_{\text {so }} \ll h \ll \epsilon_{F}$ with $\Delta_{\text {so }}=\alpha k_{F}$, as well as small exchange component of the impurity potential. Here, however, we focus on the remarkably rich behavior of the anomalous Hall conductivity beyond this special limit. Although our treatment is set up for quantum spins, we shall restrict most formulas to classical spins in the remainder of the paper. The corresponding expressions for quantum spins are similar but more cumbersome. We do, however, include the results for quantum spins in the figures.

Mechanisms.-Our model Eq. (11) contains the spinorbit interaction only in the band structure. In this sense, the AHE in this model is entirely intrinsic. Nevertheless, there are still several mechanisms which contribute to the anomalous Hall conductivity, such as the Berryphase, the skew-scattering, and the side-jump contributions. Our diagrammatic results properly capture all of these contributions. However, beyond low-order perturbation theory [15] it is difficult to disentangle the diagrammatic results. Specifically, the ladder diagrams in Fig. 1(a) contain Berry-phase and side-jump contributions. Third oder skew-scattering diagrams are collected in Fig. 1(b). Also, in the present formalism all contributions to the AHE come from the Fermi surface, while in Ref. 17] the Berry phase contribution has been attributed to the whole Fermi volume.

The skew-scattering diagrams collected in Fig. 1(b) dominate in the limit of strong spin-orbit coupling $\epsilon_{\text {so }} \gg$ $\epsilon_{J}, \epsilon_{V}$ where

$$
\sigma_{y x}^{I, s} \approx \sigma_{y x}^{I, l} \frac{\epsilon_{\mathrm{so}}\left(\epsilon_{V}^{4}-\epsilon_{J}^{4}\right)}{4 \epsilon_{J}^{2} \epsilon_{V}\left(\epsilon_{V}^{2}+3 \epsilon_{J}^{2}\right)} .
$$

Here, we introduced the energy scale $\epsilon_{V} \equiv n_{i} V$, which measures the potential disorder strength. By contrast, for weaker spin-orbit coupling $\epsilon_{\text {so }} \ll \epsilon_{J}, \epsilon_{V}$, skew scattering and the ladder contributions can be of similar order of magnitude. For this reason, our plots always refer to the sum $\sigma_{y x}^{I, l}+\sigma_{y x}^{I, s}$, cf. Figs. 2 and 3 .

Small impurity magnetization.-In the Rashba model with pointlike disorder, the AHE vanishes in the absence of magnetic scattering when both subbands are occupied [10, 12]. At small impurity magnetization we therefore expect that the anomalous Hall conductivity is proportional to the magnetization, i.e., to $h=n_{i} J\left\langle S_{z}\right\rangle$. However, in our model this holds only for very small polarization of the impurity spins. This can be understood
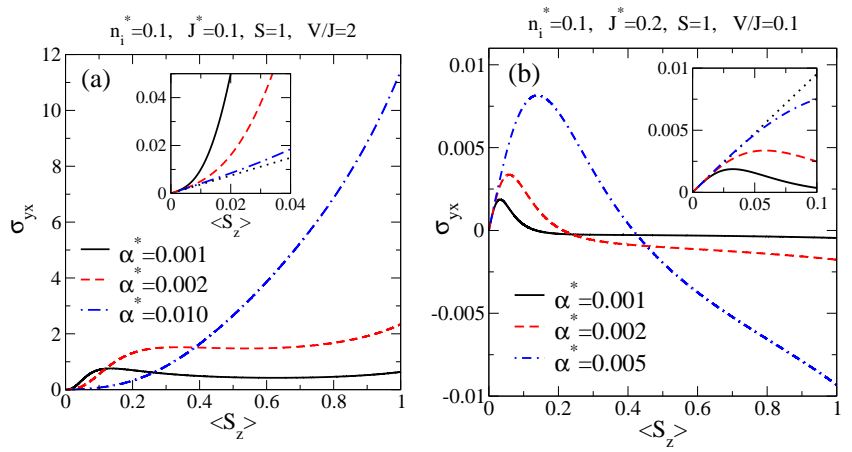

FIG. 2: (Color online.) Anomalous Hall conductivity for quantum spins with $S=1$ as function of the polarization of the impurity spins $\left\langle S_{z}\right\rangle$. The insets display zoom-ins for small impurity magnetizations, where the dotted lines indicate the $\alpha$-independent slope for small polarization $\left\langle S_{z}\right\rangle$. All conductivities are plotted in units of $e^{2} /(2 \pi)$ and we have used dimensionless units of the form $n_{i}^{*}=n_{i} / k_{F}^{2}, m^{*}=1 / 2$, $J^{*}=2 m J / \hbar^{2}, V^{*}=2 m V / \hbar^{2}, \alpha^{*}=2 m \alpha /\left(\hbar^{2} k_{F}\right)$.

analytically from the small magnetization expansion (for classical spins)

$$
\begin{aligned}
& \sigma_{y x}^{I, l} \approx \frac{e^{2}}{2 \pi} \frac{\left\langle S_{z}\right\rangle}{S} \frac{16 \epsilon_{J}^{3}}{3 \epsilon_{V}^{2}+7 \epsilon_{J}^{2}}\left(\frac{\epsilon_{V}}{\epsilon_{V}^{2}+\epsilon_{J}^{2}}-\frac{2 \epsilon_{J}^{2}}{\epsilon_{F}\left(3 \epsilon_{V}^{2}+7 \epsilon_{J}^{2}\right)}\right), \\
& \sigma_{y x}^{I, s} \approx-\frac{e^{2}}{2 \pi} \frac{\left\langle S_{z}\right\rangle}{S} \frac{18 \epsilon_{\mathrm{so}} \epsilon_{J}\left(\epsilon_{J}^{2}-\epsilon_{V}^{2}\right)}{\left(7 \epsilon_{J}^{2}+3 \epsilon_{V}^{2}\right)^{2}} .
\end{aligned}
$$

Note that a nonzero exchange coupling $J$ is required for a non-vanishing AHE. Moreover, since the skew scattering contribution $\sigma_{y x}^{I, s}$ is negligible for $\epsilon_{\mathrm{so}} \ll \epsilon_{J}, \epsilon_{V}$, we obtain the remarkable result that in this limit, the slope of the anomalous Hall conductivity becomes approximately independent of the magnitude $\alpha$ of the spin-orbit interaction. Since a finite spin-orbit coupling is required for the existence of the AHE in the first place, this implies that the regime over which the linear magnetization dependence holds, must shrink with decreasing spin-orbit coupling $\alpha$, entailing a strongly nonlinear behavior of the anomalous Hall conductivity vs. polarization of the impurity spins $\left\langle S_{z}\right\rangle$, as illustrated in Fig. 2 .

Remarkably, we find that the AHE can even change sign as a function of impurity magnetization (see Fig. 2(b)). This behavior is reminiscent of sign changes found experimentally in other systems [17, 18]. Inspecting the expressions for the slope [Eq. [11)] and for the conductivity at maximal spin polarization $\left\langle S_{z}\right\rangle=S$ [Eq. (13) below], we find that for $\epsilon_{\text {so }} \ll \epsilon_{J}, \epsilon_{V}$, where the skew scattering contribution to the slope is negligible, the conductivity as a function of $\left\langle S_{z}\right\rangle$ changes sign for scalar impurity potentials $V$ of intermediate strength, i.e., for $J S>V>J S \frac{\epsilon_{J}}{\epsilon_{F}} \frac{2 \epsilon_{V}^{2}+2 \epsilon_{J}^{2}}{3 \epsilon_{V}^{2}+7 \epsilon_{J}^{2}}$. Note also that the overall sign of the AHE depends on the sign of the exchange coupling $J$.

Large impurity magnetization.-The most intriguing behavior of the anomalous Hall conductivity occurs in the 

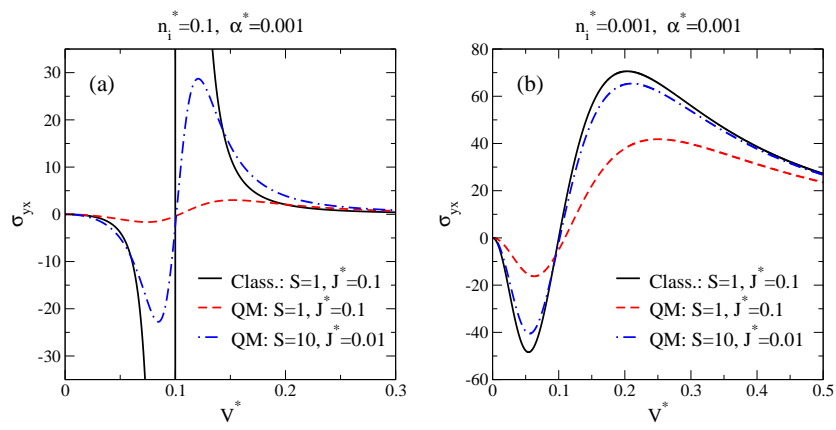

FIG. 3: (Color online.) Fully spin-polarized $\left\langle S_{z}\right\rangle=S$ anomalous Hall conductivity for classical $S=1$ spins (solid lines) and quantum spins (dashed and dashed-dotted lines) for constant $\alpha^{*}, n_{i}^{*}$ and $J^{*}$ as a function of $V^{*}$. Panel (a) shows the limit $\epsilon_{J} \gg \Delta_{\text {so }}$ and (b) the limit $\epsilon_{\text {so }} \ll \epsilon_{J} \ll \Delta_{\text {so }}$. All conductivities are plotted in units of $e^{2} /(2 \pi)$ and we have used the dimensionless units of Fig. 2

limit, where the impurity spins are near full polarization, i.e., for $\left\langle S_{z}\right\rangle \simeq S$. We first focus on the regime of weak spin-orbit coupling $\Delta_{\text {so }} \ll h=n_{i} J\left\langle S_{z}\right\rangle$ (and thus $\lambda_{F}=$ $\left.\sqrt{h^{2}+\alpha^{2} k_{F}^{2}} \approx h\right)$. In this regime, the scattering rate of the minority band

$\Gamma_{+} \approx \Gamma-\Gamma_{z} \frac{h}{\lambda_{F}}=\frac{n_{i}}{2}\left(V^{2}-2 V J\left\langle S_{z}\right\rangle \frac{h}{\lambda_{F}}+J^{2} S(S+1)\right)$

vanishes at $V=J S$ for classical spins. This absence of scattering arises from interference between the potential and magnetic components of the impurity potential and implies a divergence of the anomalous Hall conductivity. Note that this absence of scattering holds for minority spins only. For the majority band, $\Gamma_{-}$is enhanced due to the opposite sign of the interference term $\propto \Gamma_{z}$ (see Eq. (5)).

Indeed, the anomalous Hall conductivity in the regime $\Gamma \ll \epsilon_{J}, \epsilon_{V}$ for classical fully polarized magnetic impurities reads

$$
\sigma_{y x}^{I, l} \approx \frac{e^{2}}{2 \pi} \frac{4 \epsilon_{\mathrm{so}} \epsilon_{F}^{2} \epsilon_{V}^{2}\left(\epsilon_{J}^{2}+\epsilon_{V}^{2}\right)}{\epsilon_{J}\left(\epsilon_{V}^{2}-\epsilon_{J}^{2}\right)^{3}}, \sigma_{y x}^{I, s} \approx \sigma_{y x}^{I, l} \frac{2 \epsilon_{J}^{2}}{\epsilon_{J}^{2}+\epsilon_{V}^{2}} .
$$

This expression obviously diverges for $\epsilon_{V}=\epsilon_{J}$ and also implies a sign change of the anomalous Hall conductivity at $\epsilon_{V}=\epsilon_{J}$. For quantum spins the true divergence is eliminated. Nevertheless, a strong enhancement of the AHE is predicted at full spin polarization for $\epsilon_{V} \approx \epsilon_{J}$. As expected, the result for quantum spins approaches the classical divergence as the spin increases (see Fig. 3).

In addition, the divergence in the approximate expression, Eq. (13), is also cut off by a finite spin-orbit coupling $\alpha$. However, the AHE remains strongly enhanced near full polarization $\left\langle S_{z}\right\rangle=S$ even when $\epsilon_{\text {so }} \ll \epsilon_{J} \ll \Delta_{\text {so }}$. In this regime of strong spin-orbit scattering, we obtain

$$
\sigma_{y x}^{I, l} \approx \frac{e^{2}}{2 \pi} \frac{4 \epsilon_{J}^{3} \epsilon_{V}^{2}\left(\epsilon_{V}^{2}-\epsilon_{J}^{2}\right)}{\epsilon_{\mathrm{so}}\left(\epsilon_{V}^{2}+\epsilon_{J}^{2}\right)^{2}\left(\epsilon_{V}^{2}+3 \epsilon_{J}^{2}\right)}, \sigma_{y x}^{I, s} \approx \sigma_{y x}^{I, l} \frac{2 \epsilon_{J}^{2}}{\epsilon_{V}^{2}+3 \epsilon_{J}^{2}}
$$

for classical spins. Obviously, the true divergence of the anomalous Hall conductivity at $\epsilon_{V}=\epsilon_{J}$ has disappeared but a pronounced maximum of the anomalous Hall conductivity survives at $\epsilon_{V} \approx 2.2 \epsilon_{J}$ as can be seen in Fig. 3(b) (black line). Again quantum spins behave very similarly, and the classical result is recovered for large spins. Interestingly, for fully polarized quantum spins, $\left\langle S_{z}\right\rangle=S$, the anomalous Hall conductivity becomes maximal in the crossover regime between the two limits discussed above, i.e., for $\epsilon_{J} \approx \Delta_{\text {so }}$.

Conclusions.-We have investigated the effects of magnetic impurities on the anomalous Hall effect, and uncovered rich and unexpected behavior of the anomalous Hall conductivity. We find a highly nonlinear dependence on the spin magnetization, sign changes of the AHE as function of magnetization, as well as a resonant enhancement due to interference between potential and magnetic scattering from the magnetic impurities.

Although our work is motivated by the ubiquitous presence of magnetic impurities in spintronics materials, we have focused here on a thorough theoretical analysis of a simple model system, namely a two-dimensional electron system with Rashba spin-orbit coupling. A more realistic description of the band structure of real spintronics materials remains an important task for future research. Indeed, recent experimental work [18] on the diluted magnetic semiconductor (In, $\mathrm{Mn}) \mathrm{Sb}$ exhibits an intriguing sign change of the anomalous Hall conductivity as function of the impurity magnetization which is interpreted in terms of the Berry-phase contribution to the AHE [17]. Our work shows that the occurrence of such sign changes is much more generic and a conclusive interpretation of the experimental data must await more detailed research.

Acknowledgments. - This work was supported by a MÖB-DAAD grant as well as SPP 1285 of the DFG. G.Z. Has been supported by the Hungarian Grants OTKA T046303 and NF061726.

[1] R. Karplus and J. Luttinger, Phys. Rev. 95, 1154 (1954).

[2] J. Smit, Physica 21, 877 (1957).

[3] L. Berger, Phys. Rev. B 2, 4559 (1970).

[4] For a recent review see P. Wölfle and K.A. Muttalib, Ann. Phys. 15, 508 (2006).

[5] A. Fert and A. Friederich, Phys. Rev. B 13, 397 (1976).

[6] D. Culcer, A. MacDonald, and Q. Niu, Phys. Rev. B 68, 045327 (2003).

[7] V.K. Dugaev et al., Phys. Rev. B 71, 224423 (2005).

[8] N.A. Sinitsyn et al., Phys. Rev. B 72, 045346 (2005).

[9] S. Onoda, N. Sugimoto, and N. Nagaosa, Phys. Rev. Lett. 97, 126602 (2006).

[10] J.-i. Inoue et al., Phys. Rev. Lett. 97, 046604 (2006); T. Kato et al., New J. of Phys. 9, 350 (2007).

[11] M.F. Borunda et al., Phys. Rev. Lett. 99, 066604 (2007).

[12] T.S. Nunner et al., arXiv:0706.0056 
[13] I. Smorchkova and N. Samarth, Appl. Phys. Lett. 69, 1640 (1996).

[14] J. Cumings et al., Phys. Rev. Lett. 96, 196404 (2006).

[15] N.A. Sinitsyn et al., Phys. Rev. B 75, 045315 (2007).

[16] P. Streda, J. Phys. C 15, L717 (1982).
[17] T. Jungwirth, Q. Niu, and A.H. MacDonald, Phys. Rev. Lett. 88, 207208 (2002).

[18] G. Mihály et al., arXiv:0709.0059. 